\title{
PRIMARY DECOMPOSITION AND SECONDARY REPRESENTATION OF MODULES
}

\author{
MASOUMEH HASANZAD AND JAFAR A'ZAMI
}

\begin{abstract}
In this paper we study the notions of primary decomposition and secondary representation of modules over a commutative ring with identity. Also we review these concepts over injective and projective modules.
\end{abstract}

\section{INTRODUCTION}

Throughout this paper, let $R$ denote a commutative ring (with identity). A submodule $N$ of an $R$-module $M$ is said to be primary if $N \neq M$ and whenever $r \in R$, $m \in M \backslash N$, and $r m \in N$, there exists a positive integer $n$ such that $r^{n} M \subseteq N$. An $R$-module $M$ is said to be secondary if $M \neq 0$ and, for each $a \in R$, the endomorphism $\varphi_{a}: M \rightarrow M$ defined by $\varphi_{a}(m)=a m$ (for $m \in M$ ) is either surjective or nilpotent. If $M$ is secondary, then $\mathfrak{p}=\operatorname{Rad}(0: M)$ is a prime ideal, and $M$ is said to be $\mathfrak{p}$-secondary. Any non-zero quotient of a $\mathfrak{p}$-secondary module is $\mathfrak{p}$-secondary.

In this paper, we shall follow Macdonald's terminology concerning secondary representation. We refer the reader to [4, 1, 3, for more details about primary decomposition and secondary representation. For each $R$-module $L$, we denote by $m \operatorname{Ass}_{R} L$ the minimal elements of the set $\operatorname{Ass}_{R} L=\left\{p \in \operatorname{Spec}(R) \mid p=0:_{R}\right.$ $x$, for some $0 \neq x \in L\}$. For each $R$-module $L$, we denote by $\operatorname{Att}_{R} L$ the set of all attached prime ideals of $L$ over the ring $R$. Also, for any ideal $\mathfrak{b}$ of $R$, the radical of $\mathfrak{b}$, denoted by $\sqrt{\mathfrak{b}}$, is defined to be the set $\left\{x \in R: x^{n} \in \mathfrak{b}\right.$ for some $\left.n \in \mathbb{N}\right\}$, and set $\Gamma_{\mathfrak{b}}(M)=\cup_{n \in \mathbb{N}}\left(0:_{M} \mathfrak{b}^{n}\right)$, the set of elements of $M$ which are annihilated by some power of $\mathfrak{b}$. Finally we denote $\{\mathfrak{p} \in \operatorname{Spec}(R): \mathfrak{p} \supseteq \mathfrak{b}\}$ by $V(\mathfrak{b})$. For any unexplained notation and terminology we refer the reader to [4, 1].

\section{The RESUlts}

Lemma 2.1. Let $M$ be an $R$-module such that $m \operatorname{Ass}_{R} M=\operatorname{Ass}_{R} M=\left\{\mathfrak{p}_{1}, \ldots, \mathfrak{p}_{n}\right\}$ and $I_{j}=\bigcap_{\substack{i=1 \\ i \neq j}}^{n} \mathfrak{p}_{i}$. Then $\bigcap_{j=1}^{n} \Gamma_{I_{j}}(M)=0$ is a minimal primary decomposition for the zero module.

2010 Mathematics Subject Classification. 13D45, 14B15, $13 \mathrm{E} 05$.

Key words and phrases. Associated prime; Cohen-Macaulay ring; Symbolic power. 
Proof. It is clear that

$$
\operatorname{Ass} \frac{M}{\Gamma_{I_{j}}(M)}=\operatorname{Ass} M \backslash V\left(I_{j}\right)=\left\{\mathfrak{p}_{j}\right\} .
$$

Hence by [4, Theorem 6.6], $\Gamma_{I_{j}}(M)$ is a $\mathfrak{p}_{j}$-primary submodule of $M$. Now let $0 \neq x \in \bigcap_{j=1}^{n} \Gamma_{I_{j}}(M)$. Since $\operatorname{Ann}_{R}(x) \subseteq Z_{R}(M)=\bigcup_{i=1}^{n} \mathfrak{p}_{i}$, it follows that there exists $1 \leq j \leq n$, such that $\operatorname{Ann}_{R}(x) \subseteq \mathfrak{p}_{j}$. On the other hand, $x I_{j}^{n}=0$ for some $n \in \mathbb{N}$ and so $I_{j}^{n} \subseteq \operatorname{Ann}_{R}(x) \subseteq p_{j}$. Consequently $\bigcap_{\substack{i=1 \\ i \neq j}}^{n} \mathfrak{p}_{i}=I_{j} \subseteq \mathfrak{p}_{j}$ and this implies that $\mathfrak{p}_{i} \subseteq \mathfrak{p}_{j}$ for some $i \in \mathbb{N}$, which is a contradiction. Therefore $\bigcap_{j=1}^{n} \Gamma_{I_{j}}(M)=0$. Now by [4, Theorem 6.8], this is a minimal primary decomposition and $\Gamma_{I_{j}}(M)$, for all $1 \leq j \leq n$, are uniquely determined.

Now we want to present a direct proof for the following corollary, using Lemma 2.1.

Corollary 2.2. Let $R$ be a Cohen-Macaulay ring and $x_{1}, x_{2}, \ldots, x_{t}$ be an $R$ sequence such that $\sqrt{I}=I=\left(x_{1}, \ldots, x_{t}\right)$ and $\operatorname{Ass}_{R} \frac{R}{I}=\left\{\mathfrak{p}_{1}, \ldots, \mathfrak{p}_{k}\right\}$. Then for all $n \geq 1, I^{n}=\mathfrak{p}_{1}^{(n)} \cap \ldots \cap \mathfrak{p}_{k}^{(n)}$ is a minimal primary decomposition for $I^{n}$.

Proof. Since $\sqrt{I}=\bigcap_{j=1}^{k} \mathfrak{p}_{j}=I$, it follows that for all $s_{i} \in\left(\bigcap_{\substack{j=1 \\ j \neq i}}^{k} \mathfrak{p}_{j}\right) \backslash \mathfrak{p}_{i}, s_{i} \mathfrak{p}_{i} \subseteq \bigcap_{j=1}^{k} \mathfrak{p}_{j}=$

I. Therefore $\mathfrak{p}_{i} R_{\mathfrak{p}_{i}}=I R_{\mathfrak{p}_{i}}$ and consequently $\mathfrak{p}_{i}^{n} R_{\mathfrak{p}_{i}}=I^{n} R_{\mathfrak{p}_{i}}$ for all $n \in \mathbb{N}$. Also $R / I^{n}$ is Cohen-Macaulay by [4, Ex. 17.4], and we have the exact sequence

$$
0 \longrightarrow \frac{\mathfrak{p}_{i}^{(n)}}{I^{n}} \longrightarrow \frac{R}{I^{n}} \longrightarrow \frac{R}{\mathfrak{p}_{i}^{(n)}} \longrightarrow 0
$$

which implies that $\operatorname{Ass}_{R} \frac{\mathfrak{p}_{i}^{(n)}}{I^{n}} \subseteq$ Ass $\frac{R}{I^{n}}$. On the other hand, $\frac{\mathfrak{p}_{i}^{(n)} R_{\mathfrak{p}_{i}}}{I^{n} R_{\mathfrak{p}_{i}}}=\frac{\mathfrak{p}_{i}^{n} R_{\mathfrak{p}_{i}}}{I^{n} R_{\mathfrak{p}_{i}}}=\overline{0}$ and so $\mathfrak{p}_{i} \notin \operatorname{Ass} \frac{\mathfrak{p}_{i}^{(n)}}{I^{n}}$. Hence

$$
\operatorname{Ass}_{R} \frac{\mathfrak{p}_{i}^{(n)}}{I^{n}} \subseteq \operatorname{Ass}_{R} \frac{R}{I^{n}} \backslash\left\{\mathfrak{p}_{i}\right\}=\left\{\mathfrak{p}_{j}\right\}_{j=1}^{k} \backslash\left\{\mathfrak{p}_{i}\right\}
$$

Also $\mathfrak{p}_{j} \in \operatorname{Supp} \frac{R}{I^{n}}$ and for all $i \neq j, \mathfrak{p}_{j} \notin \operatorname{Supp}_{R} \frac{R}{\mathfrak{p}_{i}^{(n)}}$, hence $\mathfrak{p}_{j} \in \operatorname{Supp} \frac{\mathfrak{p}_{i}^{(n)}}{I^{n}}$. This implies that $\mathfrak{p}_{j} \in m$ Ass $\frac{\mathfrak{p}_{i}^{(n)}}{I^{n}}$. Therefore

$$
\left\{\mathfrak{p}_{j}\right\}_{j=1}^{k} \backslash\left\{\mathfrak{p}_{i}\right\} \subseteq \operatorname{Ass}_{R} \frac{\mathfrak{p}_{i}^{(n)}}{I^{n}} .
$$


By relations 2.2 and 2.3), we conclude that Ass $\frac{\mathfrak{p}_{i}^{(n)}}{I^{n}}=\left\{\mathfrak{p}_{j}\right\}_{j=1}^{k} \backslash\left\{\mathfrak{p}_{i}\right\}$. Now let $J_{i}:=\bigcap_{\substack{j=1 \\ j \neq i}}^{k} \mathfrak{p}_{j} ;$ then $\sqrt{\operatorname{Ann}_{R}\left(\frac{\mathfrak{p}_{i}^{(n)}}{I^{n}}\right)}=J_{i}$ and for a large $l \in \mathbb{N}$, we have $J_{i}^{l}\left(\frac{\mathfrak{p}_{i}^{(n)}}{I^{n}}\right)=0$, which implies that $\frac{\mathfrak{p}_{i}^{(n)}}{I^{n}} \subseteq \Gamma_{J_{i}}\left(\frac{R}{I^{n}}\right)$. Since $\Gamma_{J_{i}}\left(\frac{R}{\mathfrak{p}_{i}^{(n)}}\right)=0$, it follows from 2.1), $\frac{\mathfrak{p}_{i}^{(n)}}{I^{n}}=\Gamma_{J_{i}}\left(\frac{R}{I^{n}}\right)$, and so by Lemma 2.1 we obtain $\overline{0}=\bigcap_{i=1}^{k} \Gamma_{J_{i}}\left(\frac{R}{I^{n}}\right)=\frac{\mathfrak{p}_{1}^{(n)}}{I^{n}} \cap$ $\ldots \cap \frac{\mathfrak{p}_{k}^{(n)}}{I^{n}}$ which implies that $I^{n}=\bigcap_{j=1}^{k} \mathfrak{p}_{j}^{(n)}$.

Also Ass $\frac{\frac{R}{I^{n}}}{\frac{\mathfrak{p}_{i}^{(n)}}{I^{n}}}=\operatorname{Ass} \frac{\frac{R}{I^{n}}}{\Gamma_{J_{i}}\left(\frac{R}{I^{n}}\right)}=\operatorname{Ass} \frac{R}{I^{n}} \backslash V\left(J_{i}\right)=\left\{\mathfrak{p}_{i}\right\}$. This shows that Ass $\frac{R}{\mathfrak{p}_{i}^{(n)}}=$ $\left\{\mathfrak{p}_{i}\right\}$ and so $\mathfrak{p}_{i}^{(n)}$ is $p_{i}$-primary. Consequently $I^{n}=\bigcap_{i=1}^{k} \mathfrak{p}_{i}^{(n)}$ is a minimal primary decomposition.

We shall prove the following well-known theorem as a dual of Lemma 2.1 with a new proof.

Theorem 2.3 (Dual of Lemma 2.1). Let $R$ be an Artinian ring and $M$ be a nonzero finitely generated $R$-module such that $\operatorname{Att}(M)=\left\{\mathfrak{p}_{1}, \mathfrak{p}_{2}, \ldots, \mathfrak{p}_{n}\right\}$. Then $M$ has a minimal secondary representation as $M=\Gamma_{\mathfrak{p}_{1}}(M)+\Gamma_{\mathfrak{p}_{2}}(M)+\cdots+\Gamma_{\mathfrak{p}_{n}}(M)$, for $1 \leq i \leq n$, with $\Gamma_{\mathfrak{p}_{i}}(M)$ a $\mathfrak{p}_{i}$-secondary submodule of $M$.

Proof. Let $M=S_{1}+S_{2}+\cdots+S_{n}$ be a minimal secondary representation with $S_{i}$ a $\mathfrak{p}_{i}$-secondary submodule of $M$, for $1 \leq i \leq n$. We shall stablish the Theorem by showing (a) that $\mathfrak{p}_{i}=\sqrt{0:_{R} \Gamma_{\mathfrak{p}_{i}}(M)}$ for each $1 \leq i \leq n,(\mathrm{~b})$ that $\Gamma_{\mathfrak{p}_{i}}(M)$ is a secondary module, and (c) $M=\Gamma_{\mathfrak{p}_{1}}(M)+\Gamma_{\mathfrak{p}_{2}}(M)+\cdots+\Gamma_{\mathfrak{p}_{n}}(M)$.

(a) Since $\Gamma_{\mathfrak{p}_{i}}(M)$ is a finitely generated submodule of $M$, there exists $t \geq 1$ such that $\Gamma_{\mathfrak{p}_{i}}(M)=0: M \mathfrak{p}_{i}^{t}$. Thus $\mathfrak{p}_{i} \subseteq \sqrt{0:_{R} \Gamma_{\mathfrak{p}_{i}}(M)}$. It is enough to prove that $\sqrt{0:_{R} \Gamma_{\mathfrak{p}_{i}}(M)} \neq R$. Suppose this is not true; then $\Gamma_{\mathfrak{p}_{i}}(M)=0$. So $\operatorname{Supp}\left(\frac{R}{\mathfrak{p}_{i}^{t}}\right) \cap$ $\operatorname{Ass}_{R}(M)=\emptyset$ and hence $\mathfrak{p}_{i} \notin \operatorname{Ass}_{R}(M)$. By [4, Exer. 6.8], $\operatorname{Ass}_{R}\left(S_{i}\right)=\left\{\mathfrak{p}_{i}\right\}$ and by the exact sequence $0 \rightarrow S_{i} \rightarrow S_{i} \oplus S_{i+1} \rightarrow S_{i+1} \rightarrow 0$ we have

$$
\begin{aligned}
\emptyset & \neq \operatorname{Ass}_{R}\left(S_{i}\right) \\
& \subseteq \operatorname{Ass}_{R}\left(S_{i} \oplus S_{i+1}\right) \\
& \subseteq \operatorname{Ass}_{R}\left(S_{i+1}\right) \cup \operatorname{Ass}_{R}\left(S_{i}\right) .
\end{aligned}
$$

Since $\operatorname{Ass}_{R}\left(S_{i} \oplus S_{i+1}\right) \subseteq \operatorname{Ass}_{R}(M)$, we have $\operatorname{Ass}_{R}\left(S_{i} \oplus S_{i+1}\right)=\mathfrak{p}_{i+1}$, which shows that $\mathfrak{p}_{i+1}=\mathfrak{p}_{i}$ and this is contradiction. 
(b) Since $\Gamma_{\mathfrak{p}_{i}}(M)$ is Noetherian and $\mathfrak{p}_{i}^{t} \cdot \Gamma_{\mathfrak{p}_{i}}(M)=0, \Gamma_{\mathfrak{p}_{i}}(M)$ is Artinian. Therefore, this module has finite length. On the other hand,

$$
\begin{aligned}
\emptyset & \neq \operatorname{Ass}_{R} \Gamma_{\mathfrak{p}_{i}}(M) \\
& =\left\{\mathfrak{p}_{i}\right\} \cap \operatorname{Ass}_{R}(M) \\
& =\left\{\mathfrak{p}_{i}\right\} .
\end{aligned}
$$

Therefore $\Gamma_{\mathfrak{p}_{i}}(M)$ is coprimary, and so $\Gamma_{\mathfrak{p}_{i}}(M)$ is a secondary module by 4 , Exer. 6.9].

(c) Let $m \in M$, so $m=s_{1}+s_{2}+\cdots+s_{n}$ for some $s_{i} \in S_{i}$ (for $1 \leq i \leq n$ ). For $1 \leq i \leq n, \mathfrak{p}_{i}^{t_{i}} \cdot S_{i}=0$ for some $t_{i} \geq 1$. Hence $\mathfrak{p}_{i}^{t_{i}} \cdot s_{i}=0$ and so $s_{i} \in \Gamma_{\mathfrak{p}_{i}}(M)$ for $1 \leq i \leq n$.

\section{InJECTIVE AND PROJECTIVE MODULES}

Lemma 3.1. Let $E$ be an injective $R$-module and let $N$ be a $\mathfrak{p}$-primary submodule of $E$. Then $\left(0:_{E}\left(N:_{R} E\right)\right)$, if non-zero, is $\mathfrak{p}$-secondary.

Proof. Let $a \in R$. If $a \in \mathfrak{p}$, then $a^{n} \in\left(N:_{R} E\right)$ for some positive integer $n$, so that $a^{n}$ annihilates $\left(0:_{E}\left(N:_{R} E\right)\right)$. On the other hand, if $a \notin \mathfrak{p}$, then we can show that $a\left(0:_{E}\left(N:_{R} E\right)\right)=\left(0:_{E}\left(N:_{R} E\right)\right)$.

Let $x \in\left(0:_{E}\left(N:_{R} E\right)\right)$. There is a well-known homomorphism $\varphi: \frac{R}{\left(N:_{R} E\right)} \longrightarrow$ $E$ for which $\varphi\left(b+\left(N:_{R} E\right)\right)=b x$ for all $b \in R$. Multiplication by $a$ on $\frac{R}{\left(N:_{R} E\right)}$ is a monomorphism. So the diagram

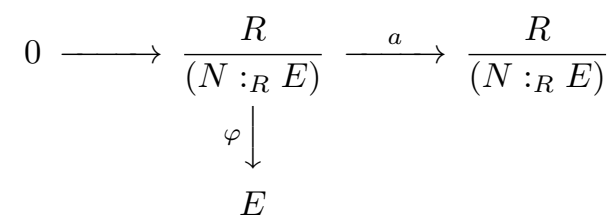

can be completed with a homomorphism $\psi: \frac{R}{\left(N:_{R} E\right)} \longrightarrow E$ which makes the extended diagram commute. Thus

$$
\begin{aligned}
x & =\varphi\left(1+\left(N:_{R} E\right)\right. \\
& =\psi\left(a+\left(N:_{R} E\right)\right) \\
& =\psi\left(a\left(1+\left(N:_{R} E\right)\right)\right. \\
& =a \psi\left(1+\left(N:_{R} E\right)\right) .
\end{aligned}
$$

Hence $x \in a\left(0:_{E}\left(N:_{R} E\right)\right)$, because

$$
\left(N:_{R} E\right) \psi\left(1+\left(N:_{R} E\right)\right)=\psi\left(N:_{R} E\right)=0_{E}
$$

this implies that $\psi\left(1+\left(N:_{R} E\right)\right) \in\left(0:_{E}\left(N:_{R} E\right)\right)$ and the proof is complete. 
Lemma 3.2. Let $I_{1}, I_{2}, \ldots, I_{n}$ be ideals of $R$ and $E$ an injective $R$-module. Then

$$
\sum_{i=1}^{n}\left(0:_{E} I_{i}\right)=\left(0:_{E} \bigcap_{i=1}^{n} I_{i}\right) .
$$

Proof. See [5, Lemma 2.2].

We recall that an injective $R$-module $E$ is said to be an injective cogenerator of $R$ if, for every $R$-module $M$ and every non-zero $m \in M$, there is a homomorphism $\varphi: M \rightarrow E$ such that $\varphi(m) \neq 0$.

Theorem 3.3. Let $E$ be an injective Noetherian $R$-module. Then $E$ has a secondary representation, and $\operatorname{Att}(E) \subseteq \operatorname{Ass}(E)$. More precisely, let $0=N_{1} \cap N_{2} \cap$ $\cdots \cap N_{n}$ be a minimal primary decomposition for the zero submodule of $E$, with $N_{i}$ $a \mathfrak{p}_{i}$-primary submodule of $E$, for $i=1,2, \ldots, n$. Then

$$
E=\left(0:_{E}\left(N_{1}:_{R} E\right)\right)+\left(0:_{E}\left(N_{2}:_{R} E\right)\right)+\cdots+\left(0:_{E}\left(N_{n}:_{R} E\right)\right)
$$

and for $i=1,2, \ldots, n,\left(0:_{E}\left(N_{i}:_{R} E\right)\right)$ is either zero or $\mathfrak{p}_{i}$-secondary.

Moreover, if $j$ is an integer such that $1 \leq j \leq n$, and $J=\{1, \ldots, j-1, j+$ $1, \ldots, n\}$, then $E=\sum_{i \in J}\left(0:_{E}\left(N_{i}:_{R} E\right)\right)$ if and only if $\bigcap_{i \in J}\left(N_{i}:_{R} E\right)$ annihilates $E$. Consequently, if $E$ is an injective cogenerator of $R$, then (*) is a minimal secondary representation for $E$, and $\operatorname{Att}(E)=\operatorname{Ass}(E)$.

Proof. By Lemma 3.1, $\left(0:_{E}\left(N_{i}:_{R} E\right)\right)$ is either zero or $\mathfrak{p}_{i}$-secondary. Generally, $\left(0:_{R} E\right)=\left(\bigcap_{i=1}^{n} N_{i}:_{R} E\right)=\bigcap_{i=1}^{n}\left(N_{i}:_{R} E\right)$. Since $\left(0:_{E} \bigcap_{i=1}^{n}\left(N_{i}:_{R} E\right)\right)=\left(0:_{E}\right.$ $\left.\left(0:_{R} E\right)\right)$, we have $e\left(0:_{R} E\right)=0$ for each $e \in E$. Thus $E \subseteq\left(0:_{E}\left(0:_{R} E\right)\right)$, and it follows that $E=\left(0:_{E} \bigcap_{i=1}^{n}\left(N_{i}:_{R} E\right)\right)$. Now, we have $E=\sum_{i=1}^{n}\left(0:_{E}\right.$ $\left.\left(N_{i}:_{R} E\right)\right)$ by Lemma 3.2 Hence $\operatorname{Att}(E) \subseteq\left\{\mathfrak{p}_{1}, \mathfrak{p}_{2}, \ldots, \mathfrak{p}_{n}\right\}$, which shows that $\operatorname{Att}(E) \subseteq \operatorname{Ass}(E)$.

For each set such as $J$, we can write:

$$
\sum_{i \in J}\left(0:_{E}\left(N_{i}:_{R} E\right)\right)=\left(0:_{E} \bigcap_{i \in J}\left(N_{i}:_{R} E\right)\right) .
$$

Now, if $\bigcap_{i \in J}\left(N_{i}:_{R} E\right)$ annihilates $E$, then $E \subseteq \sum_{i \in J}\left(0:_{E}\left(N_{i}:_{R} E\right)\right)$ and so clearly $E=\sum_{i \in J}\left(0:_{E}\left(N_{i}:_{R} E\right)\right)$ if and only if $\bigcap_{i \in J}\left(N_{i}:_{R} E\right)$ annihilates $E$.

Now, let $E$ be an injective cogenerator of $R$. It is enough to show that, for each $j=1, \ldots, n$, the ideal $\left.\bigcap_{i \in J}\left(N_{i}:_{R} E\right)\right)$ does not annihilate $E$. To prove this, let $I$ be a non-zero arbitrary ideal of $R$, and $y \in I$. Since $E$ is an injective cogenerator of $R$, there exists a homomorphism $\varphi: R \longrightarrow E$ such that $\varphi(y) \neq 0$. Hence $0 \neq \varphi(y)=y \varphi(1)$. Then $\varphi(1)$ is an element of $E$ which is not annihilated by $y$, and so not annihilated by $I$. Thus $E$ is not annihilated by $\left.\bigcap_{i \in J}\left(N_{i}:_{R} E\right)\right)$ and so $E=$ $\sum_{i=1}^{n}\left(0:_{E}\left(N_{i}:_{R} E\right)\right)$, which shows that $\operatorname{Att}(E)=\left\{\mathfrak{p}_{1}, \mathfrak{p}_{2}, \ldots, \mathfrak{p}_{n}\right\}=\operatorname{Ass}(E)$.

Remark 3.4. If $E$ is an injective module over the Noetherian ring $R$, then there is a family $\left\{\mathfrak{p}_{\alpha}\right\}_{\alpha \in \Lambda}$ of prime ideals of $R$ for which $E \cong \bigoplus_{\alpha \in \Lambda} E\left(\frac{R}{\mathfrak{p}_{\alpha}}\right)$, and if $\left\{\mathfrak{q}_{\beta}\right\}_{\beta \in \Phi}$ is a second family of prime ideals of $R$ for which $E \cong \bigoplus_{\beta \in \Phi} E\left(\frac{R}{\mathfrak{q}_{\beta}}\right)$, then there is a bijection $\gamma: \Lambda \rightarrow \Phi$ such that $\mathfrak{p}_{\alpha}=\mathfrak{q}_{\gamma(\alpha)}$ for all $\alpha \in \Lambda$. The set $\left\{\mathfrak{p}_{\alpha} \mid \alpha \in \Lambda\right\}$ is 
thus uniquely determined by $E$; we shall denote this set by $\operatorname{Occ}(E)$, and refer to its members as the prime ideals which occur in the direct decomposition of $E$.

Theorem 3.5. Let $E$ be an injective Noetherian $R$-module. Then

$$
\operatorname{Att}(E)=\left\{\mathfrak{p}^{\prime} \in \operatorname{Ass}(E) \mid \mathfrak{p}^{\prime} \subseteq \mathfrak{p} \text { for some } \mathfrak{p} \in \operatorname{Occ}(E)\right\} .
$$

Proof. Let $0=N_{1} \cap N_{2} \cap \cdots \cap N_{n}$ be a minimal primary decomposition for the zero submodule of $E$, with $N_{i}$ a $\mathfrak{p}_{i}$-primary submodule of $E$ (for $i=1,2, \ldots, n$ ). Then

$$
E=\left(0:_{E}\left(N_{1}:_{R} E\right)\right)+\left(0:_{E}\left(N_{2}:_{R} E\right)\right)+\cdots+\left(0:_{E}\left(N_{n}:_{R} E\right)\right),
$$

and (for $i=1,2, \ldots, n)\left(0:_{E}\left(N_{i}:_{R} E\right)\right)$ is either zero or $\mathfrak{p}_{i}$-secondary. Also, a minimal secondary representation for $E$ can be written from ${ }^{* *}$ ). For proving the Theorem, we shall show: (a) that $\left(0:_{E}\left(N_{i}:_{R} E\right)\right)=0$ for each $i$ for which $\mathfrak{p}_{i}$ is not contained in any $\mathfrak{p}$ in $\operatorname{Occ}(E)$, and (b) that if $j$ is an integer (with $1 \leq j \leq n$ ) for which $\mathfrak{p}_{j}$ is contained in some $\mathfrak{p}$ belonging to $\operatorname{Occ}(E)$, then $\sum_{i \in J}\left(0:_{E}\left(N_{i}:_{R}\right.\right.$ $E)) \neq E$, where $J=\{1, \ldots, j-1, j+1, \ldots, n\}$ so that $\left(0:_{E}\left(N_{j}:_{R} E\right)\right)$ cannot be omitted from $(* *)$.

(a) Let $i$ be an integer (with $1 \leq i \leq n$ ) such that $\mathfrak{p}_{i} \not \mathfrak{p}$ for all $\mathfrak{p} \in \operatorname{Occ}(E)$ ). If $\left\{\mathfrak{p}_{\alpha}\right\}_{\alpha \in \Lambda}$ is a family of prime ideals of $R$ for which $E=\bigoplus_{\alpha \in \Lambda} E\left(\frac{R}{\mathfrak{p}_{\alpha}}\right)$, then

$$
\begin{aligned}
\left(0:_{E}\left(N_{i}:_{R} E\right)\right) & \cong\left(0: \bigoplus_{\alpha \in \Lambda} E\left(\frac{R}{p_{\alpha}}\right)\right. \\
& \cong \bigoplus_{\alpha \in \Lambda}\left(0:_{E\left(\frac{R}{\mathfrak{p}_{\alpha}}\right)}\left(N_{i}:_{R} E\right)\right) .
\end{aligned}
$$

Now, in order to show that $\left(0:_{E}\left(N_{i}:_{R} E\right)\right)=0$, it is enough to show that $\left(0:_{E\left(\frac{R}{\mathfrak{p}}\right)}\left(N_{i}:_{R} E\right)\right)=0$ for all $\mathfrak{p} \in \operatorname{Occ}(E)$. For such a $\mathfrak{p}$, we have $\left(N_{i}:_{R} E\right) \not \subset \mathfrak{p}$, since $\mathfrak{p}_{i} \nsubseteq \mathfrak{p}$. Thus, there is $r \in\left(N_{i}:_{R} E\right) \backslash \mathfrak{p}$ such that multiplication by $r$ on $E\left(\frac{R}{\mathfrak{p}}\right)$ provides an automorphism of $E\left(\frac{R}{\mathfrak{p}}\right)$, and consequently $\left(0:_{E\left(\frac{R}{\mathfrak{p}}\right)}\left(N_{i}:_{R} E\right)\right)=0$.

(b) Assume that $j$ is an integer (with $1 \leq j \leq n$ ) for which $\mathfrak{p}_{j} \subseteq \mathfrak{p}$ for some $\mathfrak{p} \in$ $\operatorname{Occ}(E)$. Suppose that $E=\sum_{i \in J}\left(0:_{E}\left(N_{i}:_{R} E\right)\right)$ and $J$ defined as before. Then, by Theorem $3.3 E$ is annihilated by $a_{j}=\bigcap_{i \in J}\left(N_{i}:_{R} E\right)$ and so $E\left(\frac{R}{\mathfrak{p}}\right)$ is annihilated by $a_{j}$. Hence $a_{j} \subseteq \bigcap_{k=1}^{\infty} \mathfrak{p}^{(k)}$. Now $a_{j} \nsubseteq\left(N_{j}:_{R} E\right)$; let $r \in a_{j} \backslash\left(N_{j}:_{R} E\right)$. Then $r \in \bigcap_{k=1}^{\infty} \mathfrak{p}^{(k)}$. We know that if $f: R \longrightarrow R_{\mathfrak{p}}$ is a natural ring homomorphism, then $f \otimes i d_{E}: E \longrightarrow E_{\mathfrak{p}}$. Since ker $f \otimes E \subseteq \operatorname{ker}\left(f \otimes i d_{E}\right)$, for each $e \in E$ we have $r \otimes e=r e \in \operatorname{ker}\left(f \otimes i d_{E}\right)$. Thus, there exists $s \in R \backslash \mathfrak{p}$ such that $s(r e)=0 \in N_{j}$. But $s \notin \mathfrak{p}_{j}$ and $r e \notin N_{j}$, this is in contradiction with the fact that $N_{j}$ is $\mathfrak{p}_{j}$-primary. This completes the proof.

Lemma 3.6. Let $M$ be an $R$-module. Then $q M$ is a $\mathfrak{p}$-secondary submodule of $M$ for every $\mathfrak{p}$-secondary ideal $\mathfrak{q}$ of $R$.

Proof. Let $\mathfrak{q}$ be a $\mathfrak{p}$-secondary ideal of $R$ and $r \in R$. We consider two cases and prove the lemma. Case 1: $r \in \mathfrak{p}$. Then $r \in \sqrt{0:_{R} \mathfrak{q}}$ and so $r^{n} \mathfrak{q}=0$ for some $n \in \mathbb{N}$. Therefore $r^{n} \mathfrak{q} M=0$ and the result follows. Case 2: $r \notin \mathfrak{p}$. Then $r^{n} \mathfrak{q} \neq 0$ for each $n \in \mathbb{N}$, and so $r \mathfrak{q}=\mathfrak{q}$. Hence $r \mathfrak{q} M=\mathfrak{q} M$. It is clear that $\sqrt{0:_{R} \mathfrak{q} M}=\mathfrak{p}$. 
Theorem 3.7. Let $M$ be a representable projective $R$-module and let $M=S_{1}+$ $S_{2}+\cdots+S_{n}$, with $S_{i}$ a $\mathfrak{p}_{i}$-secondary submodule (for $i=1,2, \ldots, n$ ), be a minimal secondary representation of $M$. Then

$$
0_{R}=\left(0:_{R} S_{1}\right) \cap\left(0:_{R} S_{2}\right) \cap \cdots \cap\left(0:_{R} S_{n}\right)
$$

is a primary decomposition for the zero ideal of $R$.

Proof. We can assume that $R$ is local. Hence $M$ is a free module and so $\operatorname{Ann}_{R} M=$ 0 . Therefore the result follows.

Lemma 3.8. Let $M$ be a projective $R$-module. Then either $\mathfrak{q} M=M$ or $\mathfrak{q} M$ is a $\mathfrak{p}$-primary submodule of $M$ for every $\mathfrak{p}$-primary ideal $\mathfrak{q}$ of $R$.

Proof. See [2, Theorem 2.2].

Theorem 3.9. Let $M$ be a representable projective module over an integral domain $R$ and let $M=S_{1}+S_{2}+\cdots+S_{n}$, with $S_{i}$ a $\mathfrak{p}_{i}$-secondary submodule (for $i=$ $1,2, \ldots, n)$, be a minimal secondary representation of $M$. Also, let $\left(0:_{R} S_{i}\right) M \neq M$ for $i=1,2, \ldots, n$. Then

$$
0_{M}=\left(0:_{R} S_{1}\right) M \cap\left(0:_{R} S_{2}\right) M \cap \cdots \cap\left(0:_{R} S_{n}\right) M
$$

is a primary decomposition for the zero submodule of $M$.

Proof. Clearly, $\left(0:_{R} S_{i}\right)$, for $i=1,2, \ldots, n$, is a $\mathfrak{p}_{i}$-primary ideal of $R$. Since $M$ is projective, $\left(0:_{R} S_{i}\right) M$ will be a $\mathfrak{p}_{i}$-primary submodule of $M$, by Lemma 3.8. Let $x \in\left(0:_{R} S_{1}\right) M \cap\left(0:_{R} S_{2}\right) M \cap \cdots \cap\left(0:_{R} S_{n}\right) M$. So $x$ will be expressed as a finite sum $x=\sum_{t=1}^{k} r_{t} m_{t}$ for $r_{t} \in\left(0:_{R} S_{1}\right)$ and $m_{t} \in M$. In order to show that $x=0$, it is enough to consider $x=r_{1} m_{1}$ for $r_{1} \in\left(0:_{R} S_{1}\right)$ and $m_{1} \in M$.

If $r_{1} \in\left(0:_{R} S_{i}\right)$ for $i=2,3, \ldots, n$, then by Theorem 3.7 $r_{1}=0$ and so $x=0$. Now, let $r_{1} \notin\left(0:_{R} S_{j}\right)$ for some $1 \leq j \leq n$. So, there are two cases, $r_{1} \notin \mathfrak{p}_{j}$ or $r_{1} \in \mathfrak{p}_{j}$. If $r_{1} \notin \mathfrak{p}_{j}$, then $m_{1} \in\left(0:_{R} S_{j}\right) M$, since $r_{1} m_{1} \in\left(0:_{R} S_{j}\right) M$. Therefore $m_{1}=t m$ for some $t \in\left(0:_{R} S_{j}\right)$ and $m \in M$. Then $x=r_{1} m_{1}=r_{1} t m=0$. But, if $r_{1} \in \mathfrak{p}_{j}$, we can assume that $r_{1} \in \mathfrak{p}_{i}$ for each $i=2,3, \ldots, n$. Hence there is $k \geq 1$ such that $r_{1}^{k} \in\left(0:_{R} S_{i}\right)$ for $i=1,2, \ldots, n$. So $r_{1}^{k}=0$ by Theorem 3.7 and consequently $r_{1}=0$.

\section{REFERENCES}

[1] M. F. Atiyah and I. G. Macdonald, Introduction to commutative algebra. Addison-Wesley, 1969. MR 0242802

[2] M. Alkan and Y. Tiraş, Projective modules and prime submodules, Czechoslovak Math. J. 56 (2006), no. 2, 601-611. MR 2291760

[3] I. G. Macdonald, Secondary representation of modules over a commutative ring. In: Symposia Mathematica, Vol. XI (Convegno di Algebra Commutativa, INDAM, Rome, 1971), pp. 23-43. Academic Press, London, 1973. MR 0342506

[4] H. Matsumura, Commutative ring theory, Cambridge University Press, 1986. MR 0879273

[5] R. Y. Sharp, Secondary representations for injective modules over commutative Noetherian rings, Proc. Edinburgh Math. Soc. 20 (1976), no. 2, 143-151. MR 0414538. 
M. Hasanzad

Faculty of Mathematical Sciences, Department of Mathematics, University of Mohaghegh Ardabili, 56199-11367, Ardabil, Iran

hasanzad.masoumeh@gmail.com

J. $A^{\prime} z a m i{ }^{凶}$

Faculty of Mathematical Sciences, Department of Mathematics, University of Mohaghegh Ardabili, 56199-11367, Ardabil, Iran

jafar.azami@gmail.com, azami@uma.ac.ir

Received: March 26, 2018

Accepted: July 3, 2018 